CLINICAL STUDY

\title{
Thyroid autoimmunity in patients with malignant and benign breast diseases before surgery
}

\author{
E Giustarini, A Pinchera, P Fierabracci, M Roncella ${ }^{1}, \mathrm{~L}_{\text {Fustaino }}{ }^{1}, \mathrm{C}$ Mammoli $^{1}$ and C Giani \\ Department of Endocrinology and Metabolism, University of Pisa, Pisa, Italy, and ${ }^{1}$ Department of Surgery, Unit of Senology, S.Chiara's Hospital, Pisa, Italy \\ (Correspondence should be addressed to E Giustarini; Email: eligiustarini@hotmail.com)
}

\begin{abstract}
Background: Previous studies have demonstrated a high prevalence of thyroperoxidase antibodies (TPOAb) and autoimmune hypothyroidism in breast cancer (BC). These studies have been performed in $\mathrm{BC}$ patients generally 20-30 days after mastectomy. It is known that stress may have an influence on the immune system and a relation between stressful events and the onset or worsening of autoimmune thyroid disorders has been reported by several authors. The aim of the study was to evaluate the prevalence of autoimmune thyroid disease in patients with nodular breast disease selected for surgery before any treatment. Our hypothesis was that the high prevalence of thyroid autoimmune disorders in $\mathrm{BC}$ is independent of stressful events represented by surgery and/or anaesthetic procedures.

Methods: Our series included 61 consecutive women aged $52.8 \pm 10.2$ yrs (mean age \pm s.D.) with nodular breast disease selected for breast surgery: 36 out of 61 of them (59\%) had BC and 25 out of 61 had benign breast disease (BBD). Controls included 100 healthy age-matched women. All patients and control subjects were submitted to clinical, ultrasound thyroid evaluation and serum-free thyroxine (FT4), serum-free tri-iodothyronine (FT3), TSH, TPOAb and thyroglobulin antibodies (TgAb) determination. Results: Mean FT3, FT4 and TSH concentration showed no differences between BC patients, BBD patients and controls. The prevalence of TPOAb in BC patients $(12 / 36: 33.33 \%)$ was significantly higher than in BBD patients $(5 / 25: 20 \%)(P<0.01)$ and in controls $(8 / 100: 8 \%)(P<0.01)$. Similarly, the prevalence of $\mathrm{TgAb}$ in $\mathrm{BC}$ patients was 12 out of 36 (33.33\%) significantly higher than that detected in BBD patients $(4 / 25: 16 \%)(P<0.01)$ and in controls $(12 / 100: 12 \%)(P<0.01)$. Of the $36 \mathrm{BC}$ patients, 20 showed a diffuse hypoechogenicity of the thyroid gland to ultrasound evaluation, significantly higher than in BBD $(7 / 25: 28 \%)(P=0.03)$. Of the $20 \mathrm{BC}$ patients who showed a hypoechogenic pattern of thyroid gland, 10 $(50 \%)$ were associated with antithyroid antibodies positivity $(\mathrm{TAb})$. This finding was present in two of seven BBD $(28.57 \%)(P<0.0001)$. Only two controls showed focal hypoechogenicity of the thyroid gland. Generally, 24 out of $36(66.7 \%)$ of BC and 9 out of $25(36 \%)$ of $\mathrm{BBD}(P=0.02)$ had signs of thyroid autoimmunity consistent with the hypoechogenic pattern of thyroid gland associated or not with TAb; 2 out of $36(5.55 \%)$ of $\mathrm{BC}$ and 1 out of $25(4 \%)$ of $\mathrm{BBD}$ patients had autoimmune hypothyroidism and no hypothyroidism was found in controls.

Conclusions: The results of this study confirm the strong relation between thyroid autoimmunity and BC. This finding is independent of stressful events represented by surgery or anaesthetic procedures. The present data call attention to the usefulness of screening for autoimmune thyroid disorders in patients with nodular breast disease selected for surgery.
\end{abstract}

European Journal of Endocrinology 154 645-649

\section{Introduction}

Several prospective studies provide evidence on the relationship between breast cancer (BC) and autoimmune thyroid disorders (1-3). In particular, high prevalence of thyroperoxidase antibodies (TPOAb) and of autoimmune hypothyroidism was found in treated $\mathrm{BC}(1,2)$ and in patients with proven benign breast diseases (BBDs) (2). All these studies were carried out in patients with $\mathrm{BC}$ at different times after surgical treatment, before beginning any adjuvant anti-tumoral therapy. It is known that stress has a profound influence on the immune system through the neuroendocrine system $(4,5)$ and several studies have demonstrated a relationship between stress and autoimmunity and have indicated that stress may trigger or worsen autoimmune diseases (6-9). A possible association between stressful events and autoimmune thyroid diseases is controversial; some authors have demonstrated a relation between stress and Graves' disease (10-12) but others did not $(8,13$, 14). In contrast to Graves' hyperthyroidism, only a few reports (15-17) are available on a possible association 
between stressful events and autoimmune hypothyroidism and the results are inconclusive. In addition, the relevance of stress events on the appearance of serum thyroid antibodies or on the variation of their serum concentration is still unknown. The aim of the present prospective study was to evaluate the prevalence of autoimmune thyroid disorders in a group of patients with breast diseases selected for surgery, before any treatment including surgery. This was to confirm the high prevalence of autoimmune thyroid disorders in BC patients and to demonstrate that this finding is independent of stressful events represented by surgical treatment and/or anaesthetic procedures.

\section{Subjects and methods}

Our series included 61 consecutive women aged $52.8 \pm$ 10.2 yrs (mean age \pm s.D.), with nodular breast pathology suspected for malignancy, referred to the Surgical Department of Pisa's Hospital from May 2004 to March 2005 for breast surgery. After informed consent, all the patients were evaluated at the Department of Endocrinology and Metabolism of the same hospital for autoimmune thyroid disorder screening before surgical treatment. The mean latency period between the detection of breast lump and the first visit to the Surgical Department was 7 days and the patient was evaluated for thyroid autoimmunity the day after the first visit. The control group consisted of 100 healthy age-matched women living in the same borderline iodine sufficient geographic area. The body size of the patient group and the control group calculated as the body mass index (weight/(height) ${ }^{2}$ ) was similar: 20 and 19 respectively. A family history of autoimmune thyroid disorder was found in 5 out of $61(8.2 \%)$ patients with nodular breast diseases and in 4 out of $100(4.0 \%)$ control subjects. All patients and controls were subjected to clinical and ultrasonographic thyroid evaluation and venous blood samples were drawn for free thyroxine (FT4), free tri-iodothyroxine (FT3), thyrotrophin (TSH), thyroglobulin antibodies (TgAb) and TPOAb determination. FT3 and FT4 were measured by RIA, using Liso-phase Kits from TecnoGenetics (Milan, Italy). The sensitivity was $0.5 \mathrm{pg} / \mathrm{ml}$ for FT3 and $0.8 \mathrm{pg} / \mathrm{ml}$ for FT4. TSH was measured by a solid phase, two-site, fluoroimmunometric assay using a commercially available Kit (Delfia hTSH, Pharmacia, Friburgo, Germany): detection limit was $0.03 \mathrm{mcU} / \mathrm{ml}$. Normal ranges, defined as the mean \pm 2 s.D. values for healthy controls, were $2.7-5.7 \mathrm{pg} / \mathrm{ml}$ for FT3, 7$17 \mathrm{pg} / \mathrm{ml}$ for FT4, and $0.4-3.4 \mathrm{mcU} / \mathrm{ml}$ for TSH. $\mathrm{TgAb}$ and TPOAb were determined by two-step immunoenzymometric assay (AIA-PACK, Tosho Bioscience Inc., San Francisco, CA, USA). Briefly, TgAb or TPOAb present in the samples were bound to Tg or TPO antigens immobilized on the assay beads respectively. The beads were washed to remove unbound materials and then incubated with enzyme-labelled monoclonal antihuman IgG antibodies. The beads were washed again and then incubated with fluorogenic substrate. The amount of enzyme-labelled antibody bound to the beads is directly proportional to the TgAb or TPOAb in the samples. A standard curve using a range of known standard concentration was determined to measure unknown serum $\mathrm{TgAb}$ or TPOAb concentrations. Minimal detectable concentration of $\mathrm{TgAb}$ was $0.12 \mathrm{U} / \mathrm{ml}$ and of TPOAb was $0.05 \mathrm{U} / \mathrm{ml}$; these values correspond to 12 and $8 \mathrm{U} / \mathrm{ml}$ in original serum samples diluted 1:50 in a sample diluting solution. Values of $\mathrm{TgAb}>30 \mathrm{U} / \mathrm{ml}$ and TPOAb $>10 \mathrm{U} / \mathrm{ml}$ were considered positive for thyroid antibodies (TAb). Ultrasonographic evaluation of thyroid gland was performed using a commercially available real-time instrument (Aloka SSD121, Aloka Co., Tokyo, Japan) using a 7.5 MHz linear transducer. Thyroid volume was calculated according to the method of Aghini-Lombardi et al. (18) and the level of echogenicity was evaluated according to the method of Marcocci et al. (19). The diagnosis of autoimmune thyroid disease was carried out according to clinical, hormonal and instrumental parameters; in particular, the presence of serum TAb and/or diffuse thyroid hypoechogenicity associated or not with goitre was indicative of autoimmune thyroiditis.

\section{Statistical analysis}

Statistical analysis was performed using Fisher's exact test. A $P$ value of $<0.05$ was considered significant.

\section{Results}

Thirty-six of sixty-one (59\%) patients submitted to breast surgery for suspected nodular disease had BC, and 25 out of 61 (41\%) had BBD. In the BC group the mean \pm s.D. of serum FT4 and serum FT3 concentration was $9.76 \pm 2.73$ and $3.58 \pm 0.7 \mathrm{pg} / \mathrm{ml}$ respectively, similar to those detected in both the BBD and control groups (FT4: $9.93 \pm 2.12$ and $9.9 \pm 2.4 \mathrm{pg} / \mathrm{ml}$ ), (FT3: $3.65 \pm 0.45$ and $3.2 \pm 0.6 \mathrm{pg} / \mathrm{ml})$ respectively. The mean TSH concentration in BC was $1.9 \pm 0.7 \mathrm{mcU} / \mathrm{ml}$ similar to those detected in both the BBD group and the control group $(1.19 \pm 0.85$ and $1.8 \pm 1.4 \mathrm{mcU} / \mathrm{ml}$ respectively).

The prevalence of TPOAb in the BC group was 12 out of $36(33.33 \%)$ significantly higher than that detected in BBD patients $(5 / 25: 20 \%)(P<0.01)$, and in controls $(8 / 100: 8 \%)(P<0.01)$. The prevalence of $\mathrm{TgAb}$ in the $\mathrm{BC}$ group was $12 / 36(33.33 \%)$, in BBD patients it was 4 out of $25(16 \%)(P<0.01)$, and in the control group it was $12 \%(P<0.01)$ (Fig. 1$)$. In the positive TPOAb group, a very high concentration of serum TPOAb was detected in 5 out of $12(41.66 \%)$ BC patients and two of them showed values $>1000 \mathrm{U} / \mathrm{ml}$; in BBD 2 out of 5 


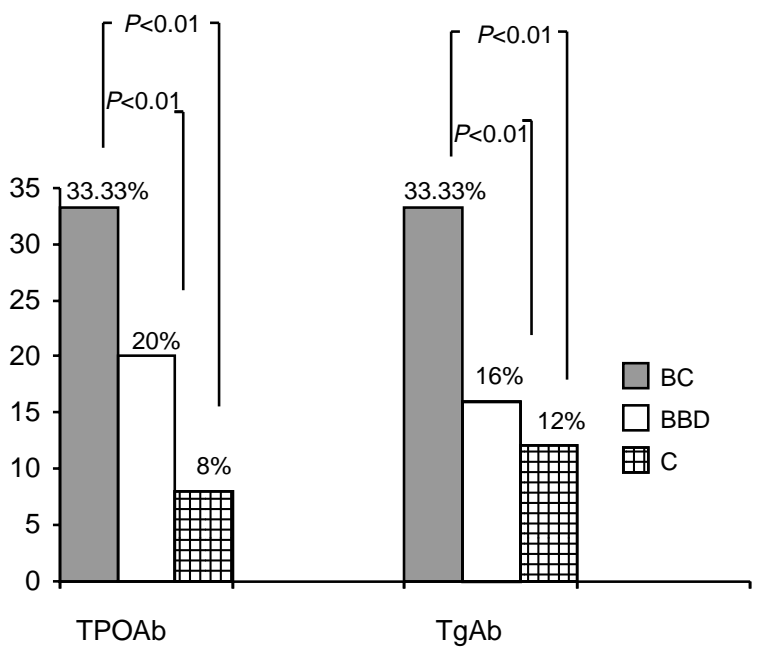

Figure 1 Distribution of TPOAb and TgAb in BC patients, BBD patients and controls (C). In BC patients, the percentage distribution of TPOAb and TgAb was significantly higher than in controls and BBD patients.

patients (40.0\%) had serum TPOAb values $>300 \mathrm{U} / \mathrm{ml}$ and only one $>1000 \mathrm{U} / \mathrm{ml}$. In the positive $\mathrm{TgAb}$ group, a very high concentration of serum $\mathrm{TgAb}(>300 \mathrm{U} / \mathrm{ml})$ was found in 8 out of $12 \mathrm{BC}$ patients $(66.66 \%)$ and in 1 out of 4 (25.0\%) BBD patients. A diffuse hypoechogenicity of thyroid gland to ultrasound evaluation was found in 20 of $36 \mathrm{BC}$ patients $(55.55 \%)$ and in 7 out of $25 \mathrm{BBD}$ patients $(28 \%)(P=0.03)$ (Fig. 2). The hypoechogenic pattern of thyroid gland was associated with $\mathrm{TAb}$ positivity in $10 \mathrm{BC}$ patients $(10 / 20: 50 \%)$ and in 2 BBD patients $(2 / 7: 28.57 \%)(P<0.0001)$ (Fig. 3). Sixteen BC patients showed a normal echographic pattern of thyroid gland and four of these (4/16: $25 \%)$ had positivity of circulant $\mathrm{TAb}$, and in BBD patients 18 out of $25(72 \%)$ had normal echographic thyroid

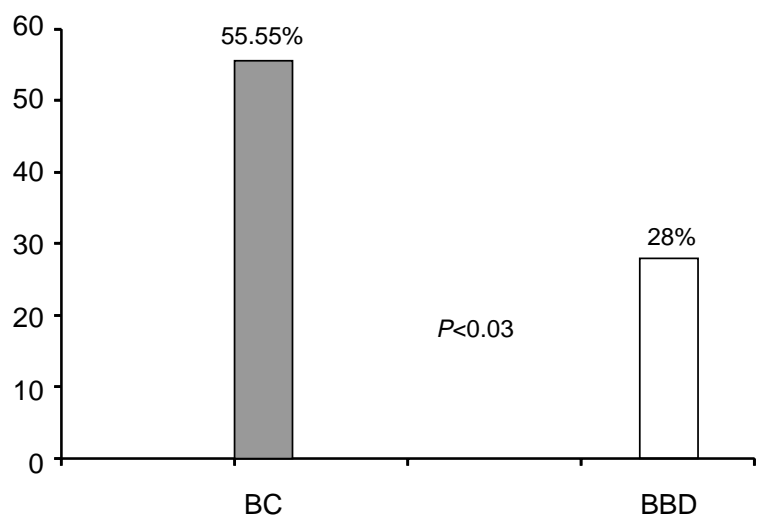

Figure 2 Frequency of hypoechogenic pattern of the thyroid gland in $\mathrm{BC}$ and $\mathrm{BBD}$ patients. Thyroid hypoechogenicity was significantly higher in the BC group.

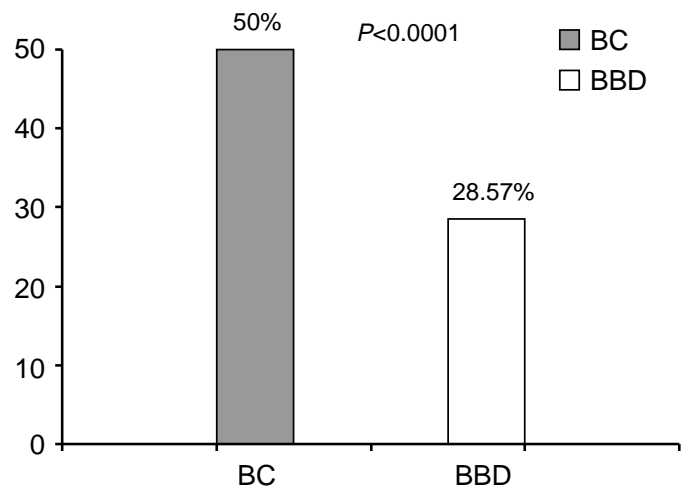

Figure 3 The percentage of hypoechogenic pattern of thyroid gland according to the presence of antithyroid antibodies in BC and BBD patients. In $B C$ patients, the percentage distribution of TAb was significantly higher than in BBD patients.

pattern with the presence of circulant antithyroid antibodies in two $(2 / 18: 11.11 \%)$. In the control group, a focal hypoechogenicity of the thyroid gland was found in only two subjects both with TPOAb positivity. In BC, 24 out of $36(66.7 \%)$ had signs of thyroid autoimmunity consistent with the hypoechogenic pattern of the thyroid gland associated or not with the presence of antithyroid antibodies and in the BBD group was 9 out of $25(36.0 \%)$. The difference was significant $(P=0.02)$ (Fig. 4). No hyperthyroidism was found in patients with breast pathology or in the control group. Two BC patients (2/36: 5.55\%) and 1 BBD patient (1/25: 4\%) had autoimmune hypothyroidism. No hypothyroidism was found in the control group.

\section{Discussion}

Various authors have focused attention on the relationship between BC and thyroid disorders (1-3). In particular, a strong relationship between breast malignancy and thyroid autoimmune disorders has been

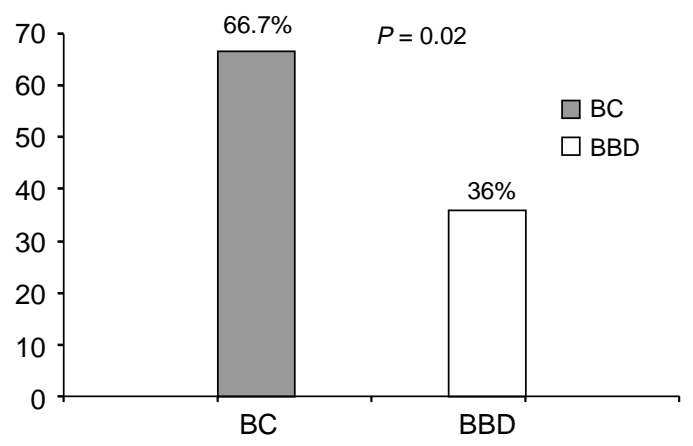

Figure 4 Frequency of thyroid autoimmunity features (hypoechogenicity of thyroid gland associated or not with antithyroid antibodies) in BC and BBD patients. The difference between the two groups was significant. 
found by Giani et al. (1) and confirmed by Smyth et al. (2). All these studies have been carried out in BC patients after mastectomy and before beginning any chemo-hormonal adjuvant therapy. The impact of stressful events on immune response and, in particular, on the onset or precipitation of autoimmune thyroid disorders is controversial. In fact, an association between stressful life events and the onset of Graves' disease has been observed in some (10-12) but not all studies $(8,13,14)$. Most of these studies were retrospective, and the use of different diagnostic criteria for thyroid diseases, the difficult definition and quantification of stressful life events and the different criteria for control groups may account for these discrepancies.

Chiovato et al. $(13,14)$, in prospective studies with well-defined criteria for thyroid autoimmune disorders in a group of patients with panic disorders and agoraphobia, showed no association between chronic recurrent stress and Graves' disease. Few studies are available on the relation between stress events and Hashimoto's thyroiditis. Two retrospective studies reported no association between lymphocytic chronic thyroiditis and stress $(16,17)$. Probably the onset and course of Hashimoto's thyroiditis are generally insidious and the patient does not become symptomatic until the development of hypothyroidism or goitre. This makes it difficult to evaluate the role of stress in the onset and/or course of these diseases. No data are available on the behaviour of thyroid antibodies after stress events.

In our study we confirmed the high prevalence of autoimmune thyroid diseases in BC. The prevalence of $\mathrm{TgAb}$ and/or TPOAb detected in the group of $\mathrm{BC}$ patients evaluated before surgery is similar to that found in a previous study in a group of $\mathrm{BC}$ patients examined after mastectomy (1). In addition, we detected a high prevalence of $\mathrm{TgAb}$ and/or TPOAb in a group of patients with BBD before surgery, significantly higher than that detected in the normal group and generally found in the normal population (20). Interestingly, a very high number of patients with $\mathrm{BC}$ had a diffuse hypoechogenicity of the thyroid gland and only half of them had antithyroid antibodies positivity. Since diffuse hypoechogenicity is a hallmark of thyroid autoimmunity, and the echographic pattern is indicative of diffuse autoimmune involvement of the thyroid gland (19) we cannot exclude the possibility that this group of $\mathrm{BC}$ patients might have autoimmune thyroiditis and develop autoimmune hypothyroidism.

In general, signs of thyroid autoimmunity, including diffuse hypoechogenicity of the thyroid gland associated or not with the presence of circulant antithyroid antibodies, were detected in the majority of $\mathrm{BC}$ patients at significantly higher levels than those detected in BBD patients. The results of this study confirm a strong relationship between thyroid autoimmunity and BC. For the first time we have demonstrated that this relationship is also present before surgery and any anti-tumoural treatment. In addition, we have demonstrated a high incidence of thyroid autoimmune disorders in BBD, but at less significant values with respect to breast malignancies. In conclusion, we confirm that all patients with breast pathology should be evaluated for thyroid autoimmune disorders, possibly to detect BC patients with hypothyroidism who need substitutive treatment before surgical treatment.

\section{References}

1 Giani C, Fierabracci P, Bonacci R, Gigliotti A, Campani D, De Negri F, Cecchetti D, Martino E \& Pinchera A. Relationship between breast cancer and thyroid disease: relevance of autoimmune thyroid disorders in breast malignancy. Journal of Clinical Endocrinology and Metabolism 199681 990-994.

2 Smyth PPA, Shering SG, Kilbane MT, Murray MJ, McDermot EWN, Smith DF \& O'Higgings NJ. Serum thyroid peroxidase autoantibodies, thyroid volume, and outcome in breast carcinoma. Journal of Clinical Endocrinology and Metabolism $1998 \mathbf{8 3}$ 2711-2716.

3 Fierabracci P, Pinchera A, Tonacchera M, Agretti P, De Marco G, Alberini S, Conforti G, Eregni E, Agresti R, Grasso L \& Giani C. Absence of interference of serum IgGs from patients with breast cancer and thyroid autoimmunity on the function of human iodide symporter gene stably transfected in $\mathrm{CHO}$ cells. Journal of Endocrinological Investigation 200427 862-865.

4 Basedovsky HO \& del Rey A. Immune-neuro-endocrine interactions: facts and hypothesis. Endocrine Reviews 199617 64-102.

5 Sternberg EM. Neuroendocrine regulation of autoimmune/inflammatory disease. Journal of Endocrinology 2001 169 429-435.

6 Barbesino G \& Chiovato L. The genetics of Hashimoto's disease. Endocrinology and Metabolism Clinics of North America 200029 357-374.

7 Strieder TG, Prummel MF, Tijessen JG, Endert E \& Wiersinga WM. Risk factors for and prevalence of thyroid disorders in a crosssectional study among healthy female relatives of patients with autoimmune thyroid disease. Clinical Endocrinology $2003 \mathbf{5 9}$ 396-401.

8 Dayan CM \& Daniels GH. Chronic autoimmune thyroiditis. New England Journal of Medicine 1996335 99-107.

9 Forsen T, Eriksson JG, Toumilehto J, Osmond C \& Barker DJ. Growth in utero and during childhood among women who develop coronary heart disease: longitudinal study. British Medical Journal $19993191403-1407$.

10 Rosh PJ. Stressful life events and Graves' disease. Lancet 1993342 566-567.

11 Paunkovic N, Paunkovic J, Pavlovic O \& Paunovic Z. The significant increase in incidence of Graves' disease in eastern Serbia during the civil war in the former Yugoslavia (1992 to 1995). Thyroid 19988 37-41.

12 Wisna B, Adami HO, Bergstrom R, Gamstedt A, Dahlberg PA, Adamson U, Jonsson R \& Karlsson A. Stressful life events and Graves' disease. Lancet 1991338 1475-1479.

13 Chiovato L \& Pinchera A. Stressful life events and Graves' disease. European Journal of Endocrinology 1996134 680-682.

14 Chiovato L, Marinò M, Pertugi G, Fiore E, Monanelli L, Lapi P, Cavaliere R, Ciampi M, Patronelli A, Placidi G, Placidi GF, Cassano GB \& Pinchera A. Chronic recurrent stress due to panic disorders does not precipitate Graves' disease. Journal of Endocrinological Investigation 199821 758-764.

15 Mizokami T, Wu Li A, El-Kaissi S \& Wall JR. Stress and thyroid autoimmunity. Thyroid 200414 1047-1055. 
16 Martin-du Pan RC. Triggering role of stress and pregnancy in the occurrence of 98 cases of Graves' disease compared to 95 cases of Hashimoto's thyroiditis and 97 cases of thyroid nodules. Annales d'endocrinologie (Paris) 199859 107-112.

17 Oretti RG, Harris B, Lazarus JH, Parkes AB \& Crownshaw T. Is there an association between life events, postnatal depression and thyroid dysfunction in thyroid antibody positive women? International Journal of Social Psychiatry 200349 70-76.

18 Aghini-Lombardi F, Pinchera A, Antonangeli L, Ragho T, Fenzi GF, Nanni P \& Vitti P. Iodized salt prophylaxis of endemic goiter: an experience in Toscana (Italy). Acta Endocrinologica 1993129 497-500.

19 Marcocci C, Vitti P, Cetani F, Catalano F, Concetti R \& Pinchera A. Thyroid ultrasonography helps to identify patients with diffuse lymphocytic thyroiditis who are prone to develop hypothyroidism. Journal of Clinical Endocrinology and Metabolism $1991 \mathbf{7 2}$ 209-213.

20 Michael W, Tunbridge G \& Vanderpump MPJ. Population screening for autoimmune thyroid disease. Endocrinology and Metabolism Clinics of North America 200029 239-253.

Received 16 November 2005

Accepted 9 December 2005 